\title{
Baseline Flight Control System for High Altitude Long Endurance Aircraft
}

\author{
Christian Weiser* \\ Institute of System Dynamics and Control, \\ German Aerospace Center (DLR), 82234 Weßling, Germany \\ Daniel Ossmann ${ }^{\dagger}$ \\ Department of Mechanical, Automotive and Aerospace Engineering, \\ Munich University of Applied Sciences HM, 80335 Munich, Germany
}

\begin{abstract}
High Altitude Long Endurance (HALE) aircraft consist of extremely light-weight structures in combination with a high wingspan and high aspect ratio. The coupling of these properties results in a dynamic behavior of the aircraft system which is different to classical transport or unmanned aircraft configurations. The key finding in the analysis of the dynamic behavior of the aeroelastic HALE aircraft is a strong interaction of structural and rigid body eigenmodes. This leads to challenges in the design of a robust flight control algorithm for the full flight envelope with state-of-the-art techniques. This work addresses these difficulties and proposes a generic design process which can be used to develop flight control algorithms for HALE aircraft. The design process starts with the definition of specific performance and robustness criteria for HALE flight control laws which emerge from the combination of general aircraft design standards with the limitations and capabilities of the HALE configuration. Subsequently, a gain-scheduled, fixed structure control design architecture is proposed. The inner loop control design is enriched with envelope protection functionalities. The design process concludes with an extensive validation and verification process to clear the baseline flight control system for flight testing. The proposed design process is applied to the German Aerospace Center's newly developed HALE platform.
\end{abstract}

\section{Introduction}

High Altitude Long Endurance (HALE) aircraft are operated at altitudes of up to $25 \mathrm{~km}$ in the stratosphere. Mission scenarios for those aircraft are similar to satellites and require the platform to stay airborne in the stratosphere from multiple days up to several months. Furthermore, the platform shall be able to fly on a desired (ground) track and perform multiple cycles from take-off to landing with the possibility of maintenance or exchange of its payload at relatively low cost. Staying airborne as heavier than air vehicle by using only solar energy requires extremely light weight structures. Compared to classical unmanned aerial vehicles, the described aspects result in high structural flexibility, a low tolerance on external (aerodynamic) loads and an unusually low airspeed in combination with a very small flight envelope. Fig. 1 1 illustrates an example design of a HALE aircraft. Historically, the design of HALE platforms has been investigated for more than 20 years [1]. First test flights, as for example of the NASA HELIOS [2], failed, because initial HALE designs were very vulnerable to atmospheric disturbances. Recent advances in solar cell and battery technology as well as composite structures, however, allow a more feasible trade-off between structural weight, aspect ratio and flexibility of the structure today. In 2018, the Airbus Zephyr aircraft made its 30-day record flight [3] which proved that the technology is actually feasible. Although several successful test flights were performed, the accident of the Zephyr in Wyndham, Australia [4] shows, that unexpected atmospheric disturbance can quickly lead to catastrophic events. Thus, there is still a gap until the HALE aircraft technology will be reliable enough for a broad band of customers, which request guaranteed availability of, e.g., surveillance or telecommunication services in the deployment region.

The flight mechanical and aeroelastic analyses of a current HALE aircraft configuration [5, 6] show, that the flight dynamics of this type of aircraft is very different from conventional UAVs in terms of eigenmodes. Especially the interaction of the structural dynamics with the short period mode is a major novel issue.

\footnotetext{
*Research Assistant, christian.weiser@dlr.de, AIAA Member

${ }^{\dagger}$ Professor, daniel.ossmann@hm.edu, AIAA Senior Member
} 


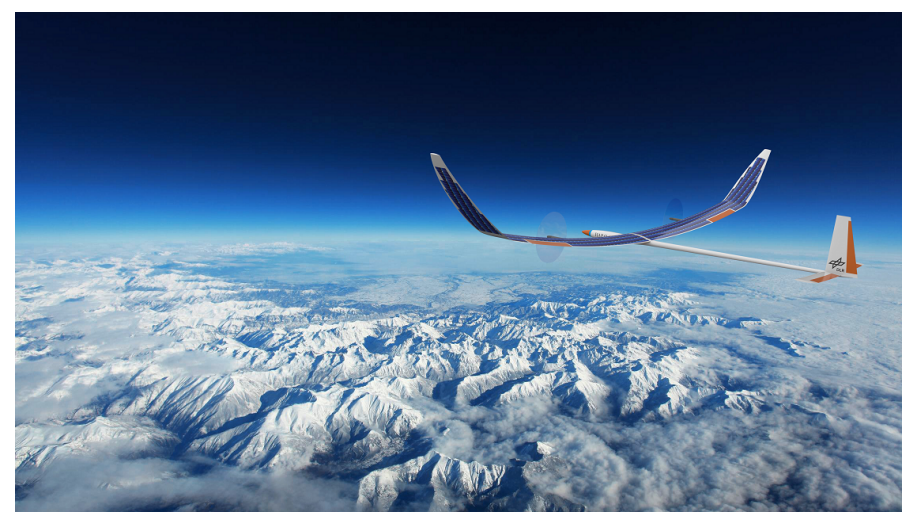

Fig. 1 Exemplary HALE aircraft representation, German Aerospace Center (DLR).

The contribution of this paper is the proposal of a generic flight control system design strategy for HALE which considers the extraordinary dynamics of the configuration, as well as the need for robustness and stability. Furthermore, implementation aspects for the software (SW) are regarded.

The first part of the paper illustrates the specific HALE aircraft dynamics using an example platform that is currently being built by the German Aerospace Center [7, 8]. The proposed flight control architecture for HALE aircraft is provided with additional insights on the design. The main part of this work describes the development of the baseline inner loop control laws which explicitly take into account the aircraft's specific flight dynamics. Based on the inner loop control design, an additional autopilot outer loop is presented. Finally, an extensive model-based verification campaign of the baseline flight control system for the given HALE aircraft proves the functionality of the closed loop aircraft system and the proposed generic design strategy.

\section{Control Architecture Definition}

The selected control architecture is depicted in Fig. 2. This paper focuses on the development of the inner and outer loop control laws for the HALE aircraft, highlighted in orange. In order to offer suitable modes for autonomous flight during nominal operation, as well as lower level modes for testing, a cascaded flight control structure is selected. In Fig. 2 and Tab. $1\left[\begin{array}{ccc}\xi & \eta & \zeta\end{array}\right]^{T}$ are the aileron, elevator and rudder deflections. $\Phi$ and $\Theta$ are the roll and pitch attitudes

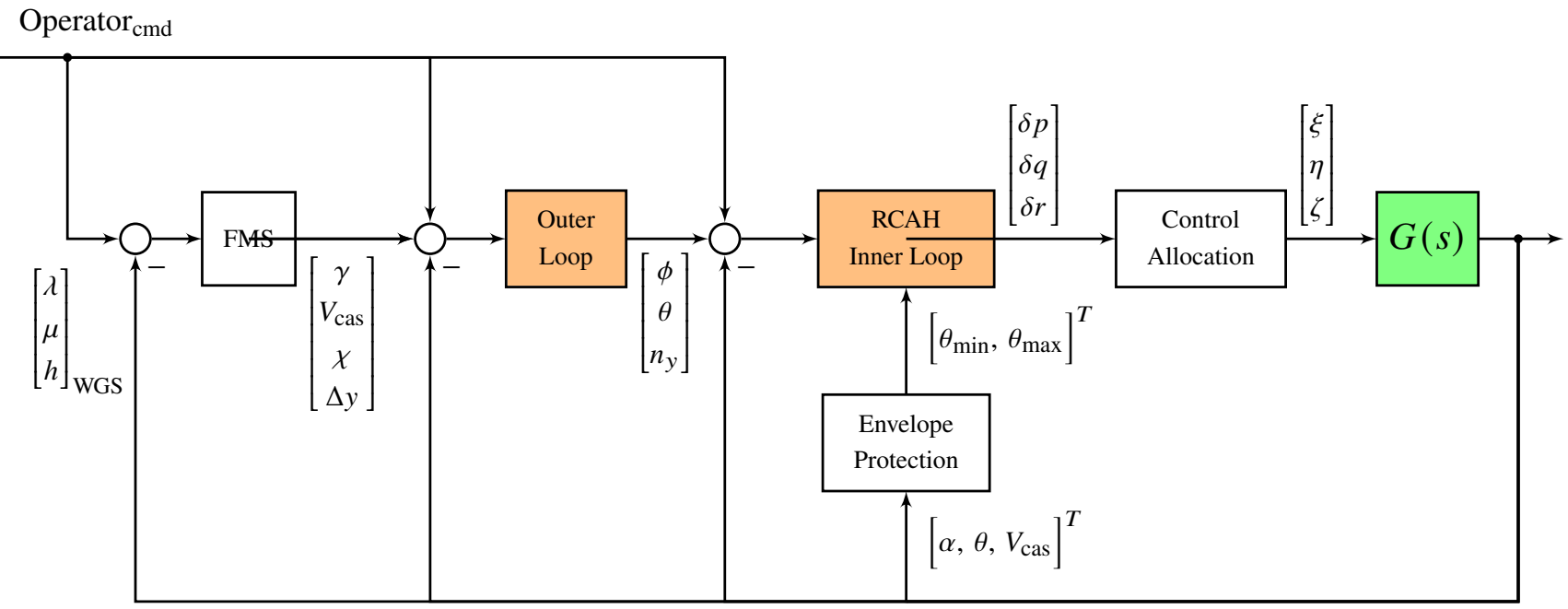

Fig. 2 Architecture of the HALE flight control system. 
and $\gamma, \chi$ represent the flight path and ground track angles. Finally, $\left[\begin{array}{lll}\lambda & \mu & h\end{array}\right]^{T}$ is the WGS position vector (longitude, latitude, altitude). The implemented modes are listed below. Further, an overview of the controlled variables for each loop is provided in Tab. 1 .

a) Manual Mode: This manual mode allows the pilot on the ground to bypass the flight control system. The only part active in the flight control computer is the mapping from the received remote-control signals to the commanded surface deflections. The pilot controls the pitch, roll and yaw axis directly via the aircraft's control surface deflections and its velocity via the thrust setting.

b) Augmented Mode: The augmented mode provides basic augmentation for the pilot. Instead of directly controlling the surfaces the pilot inputs pitch- and roll-attitude / rate commands. The lateral acceleration $n_{\mathrm{y}}$ is automatically regulated to zero, reducing the pilots need to control the yaw axis separately. Thrust remains in manual control, i.e., the pilot controls the velocity and flight path angle via the thrust setting.

c) Selected Mode: In the selected mode, an autopilot functionality is provided which ensures tracking of airspeed and flight path angle in the vertical axis, and heading / ground track in lateral axis.

d) Managed Mode: In managed mode, way-point based navigation on 2D or 3D trajectories is possible, with the flight management system computing and commanding lateral and vertical deviations from the planned flight path.

Table 1 Flight control modes.

\begin{tabular}{lcc}
\hline Mode & Longitudinal references & Lateral references \\
\hline Manual & $\eta, \mathrm{rpm}$ & $\xi, \zeta$ \\
Augmented & $\dot{\Theta}$ & $\Phi, n_{y}=0$ \\
Selected & $\mathrm{V}_{\text {eas }}, \gamma, \dot{h}$ & $\Psi / \chi$ \\
Managed & $\mathrm{V}_{\text {eas }}, \gamma, \dot{h}$ & $\chi, \Delta \mathrm{y}$ \\
\hline
\end{tabular}

\section{HALE Aircraft System Dynamics}

For modeling of the aircraft dynamics, the DLR Varloads framework [9] is used. The aerodynamics are modeled via the vortex lattice method (VLM), details on its implementation can be found in [10]. The equations of motion for the aircraft are defined as follows:

$$
\begin{aligned}
{\left[\begin{array}{c}
M_{b}\left(\dot{V}_{b}+\Omega_{b} \times V_{b}\right) \\
I_{b}\left(\dot{\Omega}_{b}+\Omega_{b} \times\left(I_{b} \Omega_{b}\right)\right.
\end{array}\right] } & =\Phi_{b a} P_{a}^{\mathrm{ext}} \\
M_{f f} \ddot{u}_{f}+D_{f f} \dot{u}_{f}+K_{f f} u_{f} & =\Phi_{f a} P_{a}^{\mathrm{ext}}
\end{aligned}
$$

with the aircraft's mass $M_{b}$ and inertia $I_{B} . V_{b}, \Omega_{b}$ in Eq. (1) denote the linear and rotational velocities of the air frame. External forces and moments $P_{a}^{\text {ext }}$ on the right hand side are calculated within the Varloads framework as described in [10], the structural dynamics model is provided by [6]. The structural dynamics Eq. (2) is a 2nd order differential equation with masses $M_{f f}$, stiffness matrix $K$, damping matrix $D$ and the displacement $u_{f}$.

The resulting non-linear flexible aircraft model is linearized at a set of operating points. The flight envelope which is considered for control design consists of two varying parameters, namely the equivalent airspeed, and altitude in flight levels $(\times 100 \mathrm{ft})$. The parameter ranges are listed in Tab. 2, where $\mathrm{V}_{\mathrm{S}}$ is the stall speed, $\mathrm{V}_{\mathrm{o}}$, $\min / \max$ are the minimum / maximum operating speed and $\mathrm{V}_{\mathrm{NE}}$ is the never exceed velocity.

Table 2 Flight envelope parameters.

\begin{tabular}{l|cccc}
\hline Parameter & \multicolumn{4}{|c}{ Values } \\
\hline $\mathrm{V}_{\text {eas }}$ & $\mathrm{V}_{\mathrm{S}}$ & $\mathrm{V}_{\mathrm{o}, \min }$ & $\mathrm{V}_{\mathrm{o}, \max }$ & $\mathrm{V}_{\mathrm{NE}}$ \\
Altitude (FL) & 0 & FL200 & FL400 & FL600 \\
\hline
\end{tabular}

The eigenvalues are displayed for the variation in airspeed from minimum to maximum at sea level and service ceiling in Figs. 3 and 4 . In Fig. 3, the short period and first symmetric bending mode of the aircraft are visible. For 


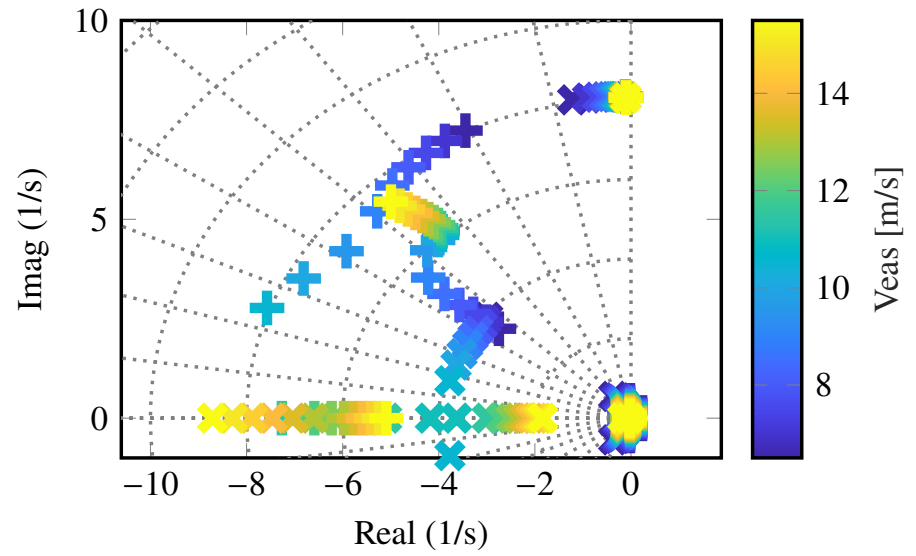

Fig. 3 Poles of the longitudinal aircraft dynamics at sea level $(x)$ and service ceiling $(+)$ for different airspeeds.

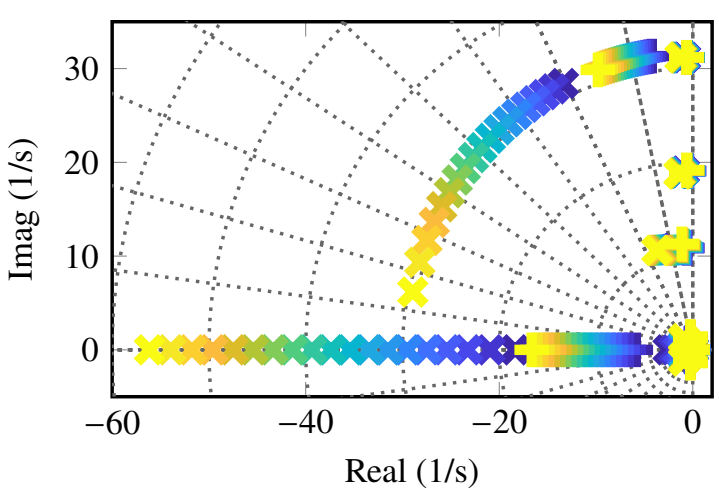

(a) Roll and low frequency structural modes.

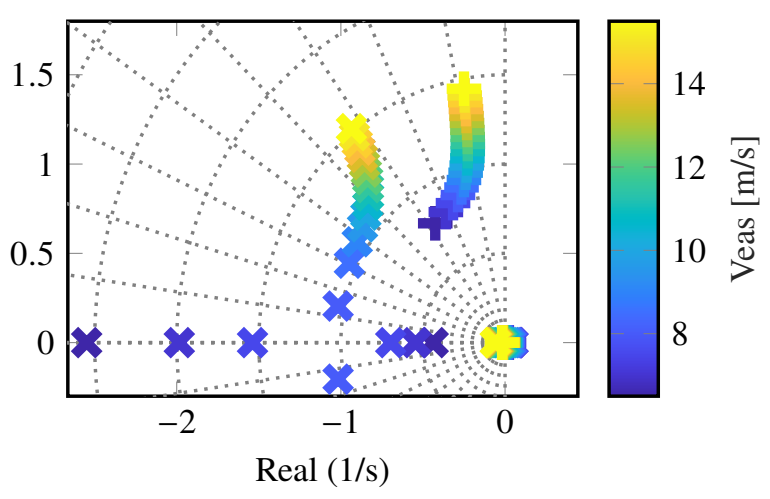

(b) Dutch roll mode.

Fig. 4 Poles of the lateral aircraft dynamics at sea level (x) and service ceiling (+) for different airspeeds.

FL0, the symmetric bending has a frequency of $4 \mathrm{rad} / \mathrm{s}$ and becomes over-damped for increasing airspeed, while the short period is not oscillating. For the service ceiling, the short period shows an almost constant damping ratio with increasing frequency from 4 to $8 \mathrm{rad} / \mathrm{s}$, while the first bending mode has a frequency of $8 \mathrm{rad} / \mathrm{s}$ and shows increasing damping again. Together with some analysis of the system's eigenvectors, it can be clearly seen that the short period and first symmetric bending mode are strongly coupled for this aircraft configuration. Thus, the design of a longitudinal control law will have the constraint of not overly destabilizing the first bending mode. The lateral axis' eigenvalues are depicted in Fig. 4a, where a similar behavior of increasingly damped structural modes of $30 \mathrm{rad} / \mathrm{s}$ can be observed. Interestingly, the roll mode has a very low time constant of as less as $1 / 55 \mathrm{~s}$, which is significantly lower in case of truncating all flexible modes. This is also a hint for strong coupling of the roll mode with some of the first flexible symmetric and asymmetric bending modes. When looking at the roll-yaw coupling, it can be seen that the dutch-roll mode damping increases for low airspeed and even becomes aperiodic at sea level. This behavior is also untypical, but can rather be explained by the unusually low airspeed and is found not to be caused by structural / rigid body couplings. Control inputs for each body axis are defined as: aileron $(\xi)$, elevator $(\eta)$ and rudder $(\zeta)$. For tuning of the control laws, states which do not influence the aircraft motion in the symmetric (for longitudinal control)/ asymmetric (lateral control) plane, are truncated. This reduces the model complexity and computational cost for control synthesis. The reduced models include the rigid body states $[\alpha, q, \Theta, V]^{T}$ as well as 10 structural modes in the longitudinal case. Fig. 5 shows the comparison of the bode magnitude plots between the full model with 6 rigid +30 flexible modes and the reduced model with 4 rigid body longitudinal states $\left([\alpha, q, \Theta, V]^{T}\right)$ and 10 flexible modes. The comparison is shown here for 2 operating points and the right hand side plot shows the model matching error $G_{\text {red }} / G_{0}$ ). It can be seen, that this matches quite well for point one, but has a $-2 \mathrm{~dB}$ offset for point 2 . This leads to the conclusion, that for achieving better results it could be beneficial to execute the model matching separately for each operating point in order to achieve 
more accurate results. For practical reasons, controller synthesis with models of the same size is preferred. Moreover, the relatively small error can be neglected and thus, the selected order reduction is carried out equally for all operating points. The selection of flexible modes which are kept within the reduced dynamics controller synthesis models is selected as the minimum number of states, which ensures a low dc-gain error and as well a minimal model matching error up to a frequency of about $2 \mathrm{rad} / \mathrm{s}$ ( $>2$ times the targeted tracking bandwidth). The results are shown in Figs. $5 \mathrm{~b}$ and 6c, where the matching errors between lower order representations and full models are depicted. Fig. 6 depicts the matching of the lateral reduced models with four rigid body states $\left([p, r, \Phi, \beta]^{T}\right)$ and 10 flexible modes to the full order model. Similar to the longitudinal case, an acceptable matching of the reduced models up to $2 \mathrm{rad} / \mathrm{s}$ is demonstrated.

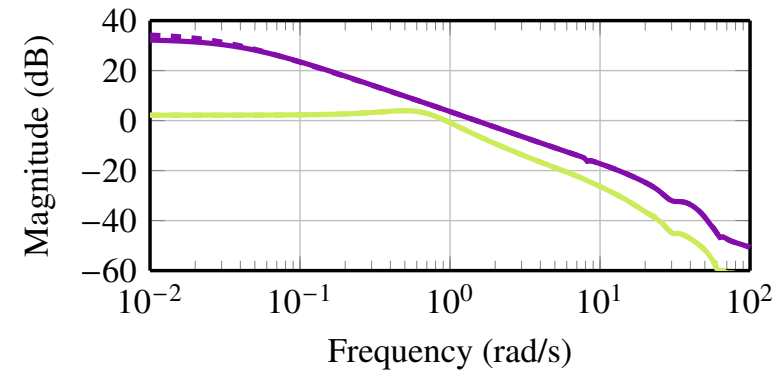

(a) Bode magnitude plot from the elevator to the pitch attitude $(\Theta)$.

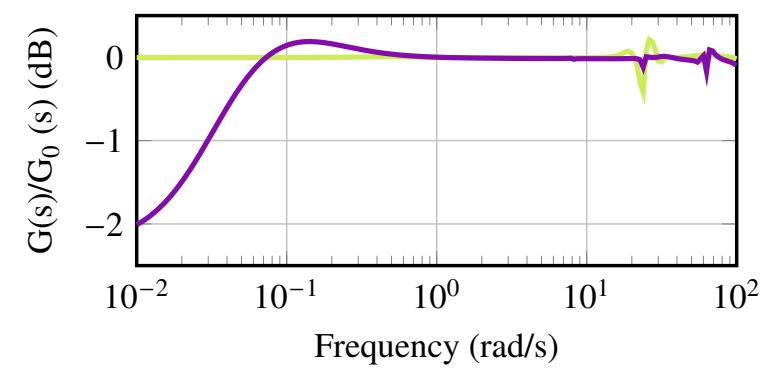

(b) Magnitude discrepancies between full order models and their approximations.

Fig. 5 Analysis of reduced order model for the longitudinal axis.

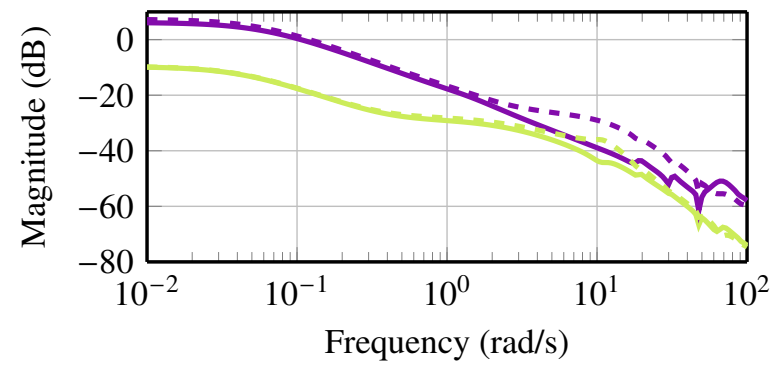

(a) Bode magnitude plot from the aileron to the bank angle $(\Phi)$.

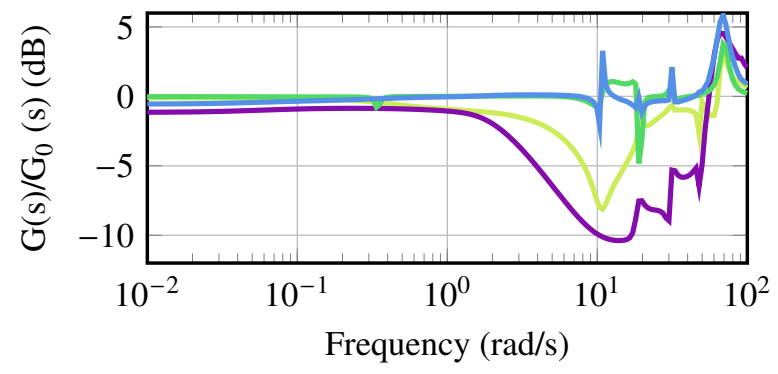

(c) Magnitude discrepancy between full order models and their approximations from Figs. 6 a and 6b.

Fig. 6 Bode magnitude responses of the lateral tracking variables for $\mathrm{min} . / \mathrm{max}$. airspeed at one selected flight level for the reduced $\square$ and full order $-=$ models.

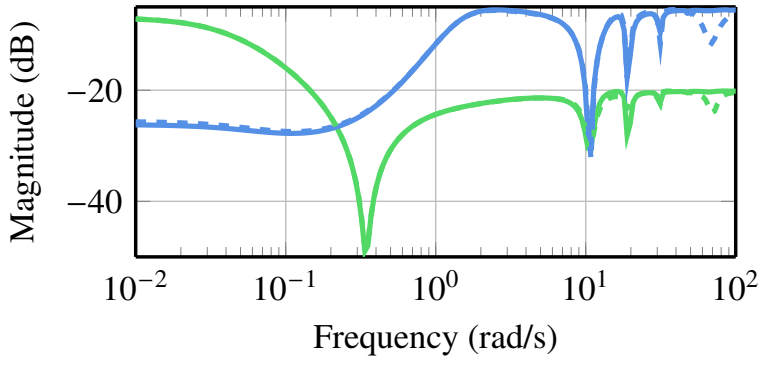

(b) Bode magnitude plot from rudder to the lateral acceleration $\left(\mathbf{N}_{y}\right)$. 


\section{Inner Loop Control Laws}

This section describes the design of the baseline inner loop control law for DLR's HALE aircraft. The control design is split into longitudinal and lateral dynamics, as the two dynamics are sufficiently decoupled. The design process for each axis follows a standardized design process similar as proposed in [11-13], including with the following three design steps:

i) Definition of closed loop requirements

ii) Selection of a suitable control architecture followed by the controller synthesis

iii) Verification of the resulting closed loop system

Step i) and ii) are discussed in detail within this section, while step iii) is discussed in section VI for a dedicated flight maneuver.

\section{A. Stability and performance requirements}

This section lists the requirements, which are to be considered during the design of the inner loop control laws. It is assumed, that functional requirements, such as provision of attitude guidance, acquisition of inputs and calculation of command signals, is provided by the overlaying loops described in the architecture illustrated in in Fig. 2

In a first step, commonly known requirements are mentioned, which the controller should fulfill:

1) Stability of the closed loop system: all closed loop eigenvalues should meet $\operatorname{Re}(\lambda)<0$.

2) Robustness : For robustness considerations, the conventional $6 \mathrm{db}$ gain margin (GM) and $45 \mathrm{deg}$ phase margin (PM) requirements should be met for any frequency. For this special case of a very flexible aircraft, this is extended to a gain margin of $8 \mathrm{db}$ and phase margin of $60 \mathrm{deg}$ for frequencies greater or equal the frequency of the first flexible aircraft mode $\left(\equiv f_{\mathrm{sp}}\right)$ [14].

3) Favorable damping ratios of e.g. the short period mode / dutch roll are defined in [14]. For the case of this HALE example, the short period mode tends to be aperiodic at many frequencies, thus this requirement is fulfilled without further action.

4) Performance specifications in accordance with refs [14, 15], concerning rise time / bandwidth evaluation of bandwidth and disturbance rejection. The performance specifications are fully enforced in the nominal operation region of the flight envelope, i.e. between $\mathrm{V}_{\text {eas }}=\left[V_{\mathrm{o}, \min }, V_{\mathrm{o}, \max }\right]$. Outside of this region, the performance criteria are relaxed.

5) The steady state error shall be sufficiently low, for the region $\left[V_{\text {stall }}, V_{\mathrm{o}, \mathrm{min}}\right]$ this specification is significantly relaxed in order not to constrain the overall optimization of the controller.

The resulting numerical criteria for the are summarized in Tab. 3 for the longitudinal controller and in Tab. 4 for the lateral controller.

Table 3 Pitch attitude controller tuning specifications.

\begin{tabular}{lcc}
\hline Name & Acceptable Value & Frequency Region \\
\hline Pitch att. bandwidth(BW) & $2 \mathrm{rad} / \mathrm{s}$ & - \\
Max. overshoot & $10 \%$ & - \\
Gain / Phase Margin & $6 \mathrm{~dB} / 45 \mathrm{deg}$ & {$[0 ; 4] \mathrm{rad} / \mathrm{s}$} \\
Gain / Phase Margin & $8 \mathrm{~dB} / 60 \mathrm{deg}$ & ] $4 ; \mathrm{inf}[\mathrm{rad} / \mathrm{s}$ \\
Dist. Rejection & $35 \mathrm{~dB}$ & {$[0 ; 0.1] \mathrm{rad} / \mathrm{s}$}
\end{tabular}

\section{B. Design}

In order to connect the functionalities of manual and automated control and provide a suitable interface for the autopilot, an Rate Command Attitude Hold (RCAH) architecture is selected. The RCAH control structure for the pitch axis is depicted in Fig. 7. It allows the pilot to directly command pitch rate, which is favorable as a neutral stick means constant pitch attitude. The integrator is part of a command filter and ensures that the pitch attitude set by the pilot is also maintained in case of disturbance. Furthermore, for the operation of the inner loop in combination with the autopilot, the attitude input from the autopilot can be directly fed to the controller, while the integrator and feed-forward $\left(K_{\mathrm{ff}}\right)$ gain can be omitted. 
Table 4 Roll attitude and turn coordination controller tuning specifications.

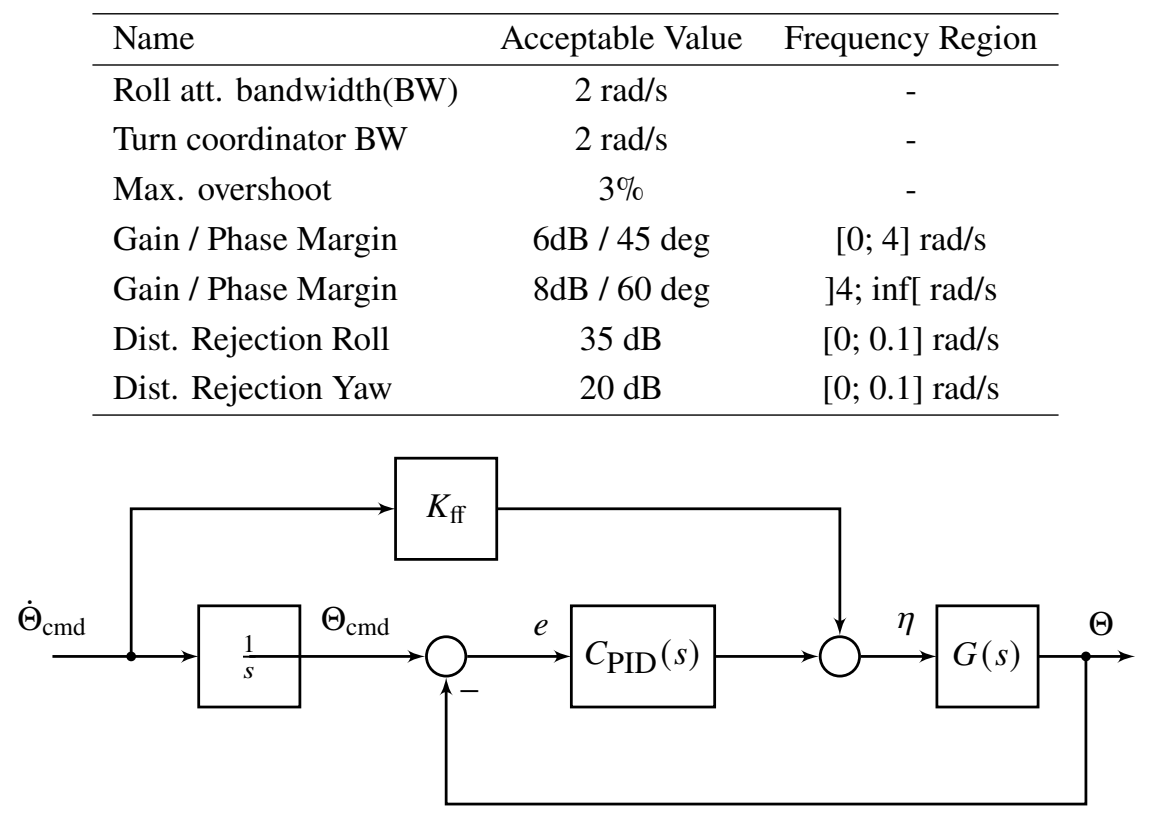

Fig. 7 Block diagram for fixed structure RCAH pitch controller.

The control law in Fig. 7 can mathematically be formulated as

$$
\eta(V, h)=K_{P}(V, h) e_{\Theta}+K_{I}(V, h) e_{\Theta}+K_{D}(V, h) \dot{\Theta}_{\text {meas }}+K_{\mathrm{ff}}(V, h) \delta \dot{\Theta}_{\mathrm{ref}}
$$

where $\eta$ is the resulting elevator command and

$$
e_{\Theta}=\delta \int \dot{\Theta}_{\mathrm{ref}} \mathrm{dt}+(1-\delta) \Theta_{\mathrm{ref}}-\Theta_{\text {meas }}
$$

the control error. The parameter $\delta$ in Eq. (3) denotes the setting of the mode switch

$$
\delta= \begin{cases}1 & \text { if "Augmented" Mode active } \\ 0 & \text { otherwise }\end{cases}
$$

where 'Augmented' refers to the scenario where the pitch autopilot supplies an attitude command. If $\delta=0$ control is executed via a pitch rate command supplied by an operator. Furthermore, gain-scheduling of the control parameters are inherently regarded in the optimization process via basis functions defined as

$$
K_{i}\left(V_{\text {eas }}, h\right)=k_{0}+k_{1} V_{\text {eas }}+k_{2} h+k_{3} V_{\text {eas }} h
$$

for each gain. The components of fixed-structure RCAH architecture described above are tuned within a constraint optimization process as, e.g., proposed in [16]:

$$
\begin{array}{rc}
\min _{K(\pi)} \max _{i, k} & f_{i}^{(k)}(K(\pi)) \\
\text { s.t. } & \max _{j, k} g_{j}^{(k)}(K(\pi))<1 \\
& K_{\min }<K(\pi)<K_{\max }
\end{array}
$$

The optimization employs the (linear) aircraft models generated in section [II], and including simplified actuator and sensor dynamics. The used optimization constraints $g$ (hard requirements) in Eq. 7 correspond to the stability requirements, whereas the minimization goals $f$ are the performance requirements which is described in section IV.A. 


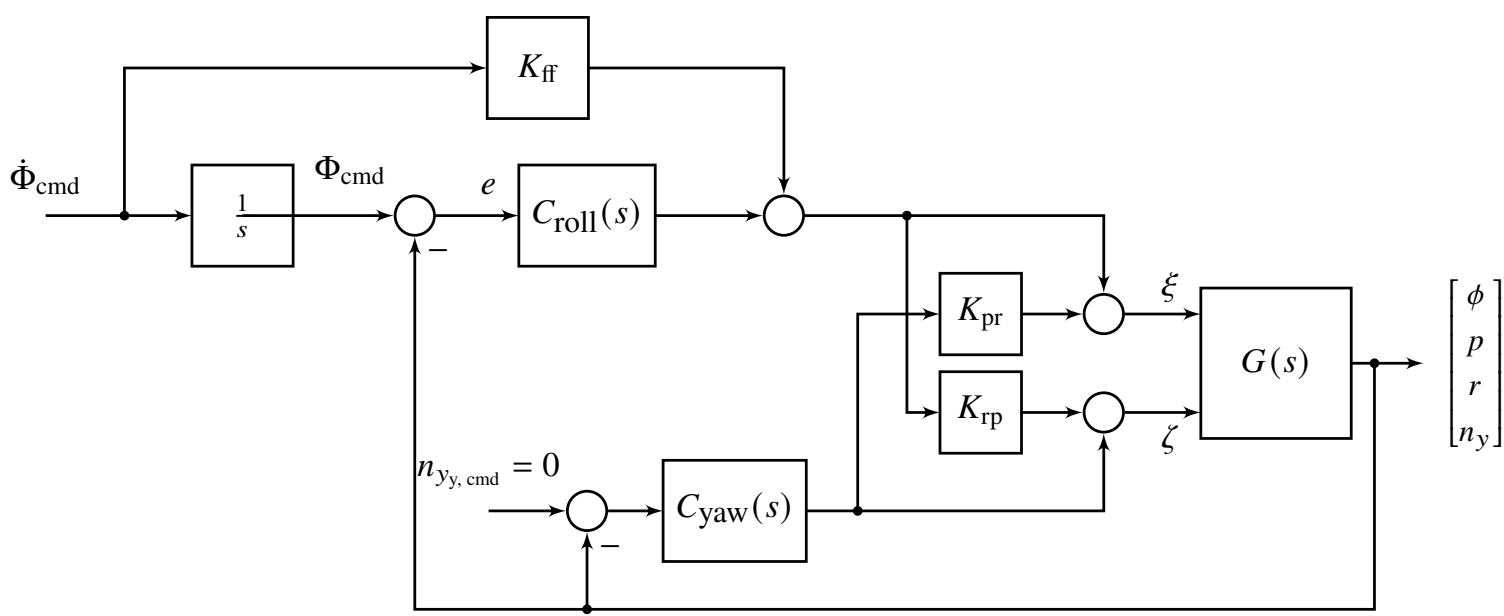

Fig. 8 Block diagram for fixed structure control design of a RCAH roll attitude controller with turn coordination by lateral acceleration feedback.

The algorithms used to solve the optimization problem in section [V.B are described in detail in [16-18] and are available as MATLAB implementations.

For lateral guidance, a similar RCAH architecture for the roll axis is selected as presented above for the pitch axis. The lateral control architecture law is depicted in Fig. 8 and allows the pilot to command a roll rate command whereas the autopilot feeds a roll attitude command. For the yaw axis, a second proportional integral derivative (PID) control structure is added, where the lateral acceleration reference $n_{\mathrm{y}, \mathrm{cmd}}$ is set to zero at all times for coordinated maneuvering via the proportional and integral gains. Yaw damping via high-pass filtered yaw rate feedback is included as well.

For development of the architecture, an eigenstructure assignment as discussed in [19, 20] is performed for linear models at selected operating points. These results show, that contrarily to lateral control for transport aircraft, the roll and yaw control should not be controlled separately, but there exists a strong cross coupling which also varies over the flight envelope parameters. Moreover, the model analysis in section III already showed that there exists this strong cross-coupling between the roll and yaw motion. As a result, the gains $K_{p r}$ and $K_{r p}$ are introduced into the control architecture to account for this effect. Afterwards, the synthesis of a scheduled control law is formulated as an optimization problem similar to the one in section IV.B with a total of eight gains to be tuned (proportional, integral gains on the two tracking variables; proportional augmentation gains on rates; two cross-coupling gains; $K_{f f}=K_{D, \Phi}$ ). The optimization goals are derived from the requirements described in section IV.A and are listed Tab. 4

\section{Synthesis Results}

The optimization results for the RCAH pitch controller are shown in Fig.9 Moreover, the result of the linear closed loop step response in Fig. 10a and the Nichols plot of the closed loop cut open at the elevator signal (Fig. 10b) are depicted. From these plots, the stability and performance requirements (Tabs. 3 and 4 ) is cross-checked in order to confirm the success of the control law synthesis. As illustrated in the last two plots of Fig. 9 and in Fig. 10b the system is stable with sufficient margins at all frequencies. Moreover, the specified rise time is met (Fig. 10a) and the specified steady tracking error criterion is met as seen in the upper plots of Fig. 9 The left hand upper plot shows the tracking criterion for nominal operation, the right upper plot depicts a relaxed criterion which demands less accurate tracking and allows more over shoot for envelope points outside the nominal operating range. For stability consideration, the lower plots of Fig. 9 show the gain and phase margin, where the nominal requirement is $6 \mathrm{~dB} / 45 \mathrm{deg}$ which is enlarged to $8 \mathrm{~dB} / 60 \mathrm{deg}$ for frequencies above $1 \mathrm{rad} / \mathrm{s}$. A further check on the stability boundaries is possible with the Nichols chart in Fig. $10 \mathrm{~b}$, where the boundaries are marked in solid red for low frequencies and dashed for frequencies above $1 \mathrm{rad} / \mathrm{s}$. A detailed $V \& V$ process is being developed for cross checking all requirements within the non-linear closed loop simulation (the detailed layout and results of the $\mathrm{V} \& \mathrm{~V}$ tool chain would go beyond the scope and will be addressed separately). Even though the available airspeed envelope is tight, it can be seen that for all three parameters the available degree of freedom to introduce a scheduling over $V_{\text {eas }}$ seems necessary. 

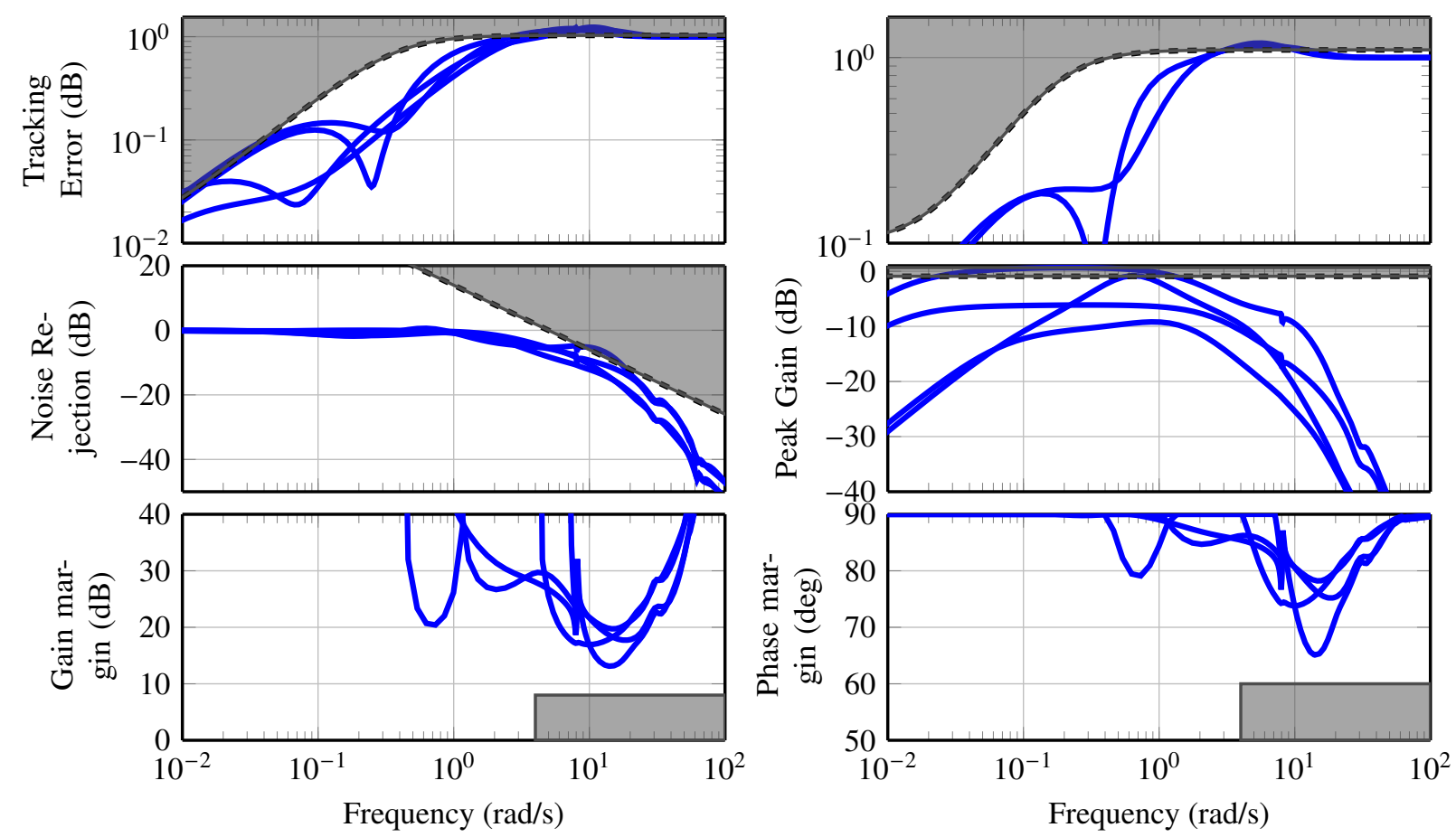

Fig. 9 Optimization results for the longitudinal control laws for the four corners of the two-parameter envelope. First row: tracking requirement, second and third row: gain/phase margins.

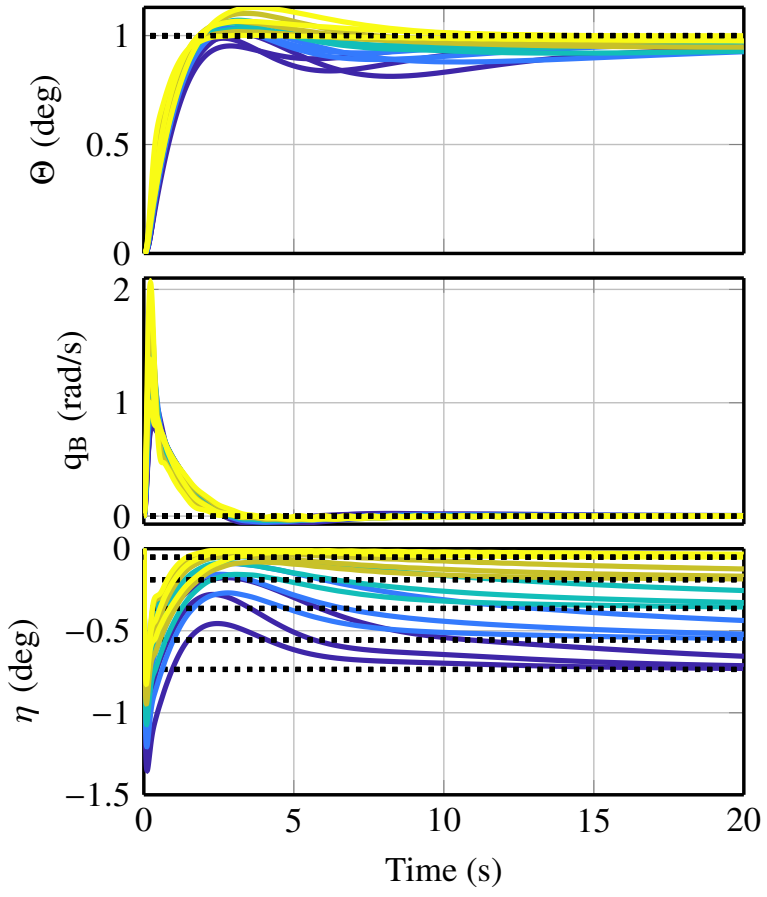

(a) Step response.

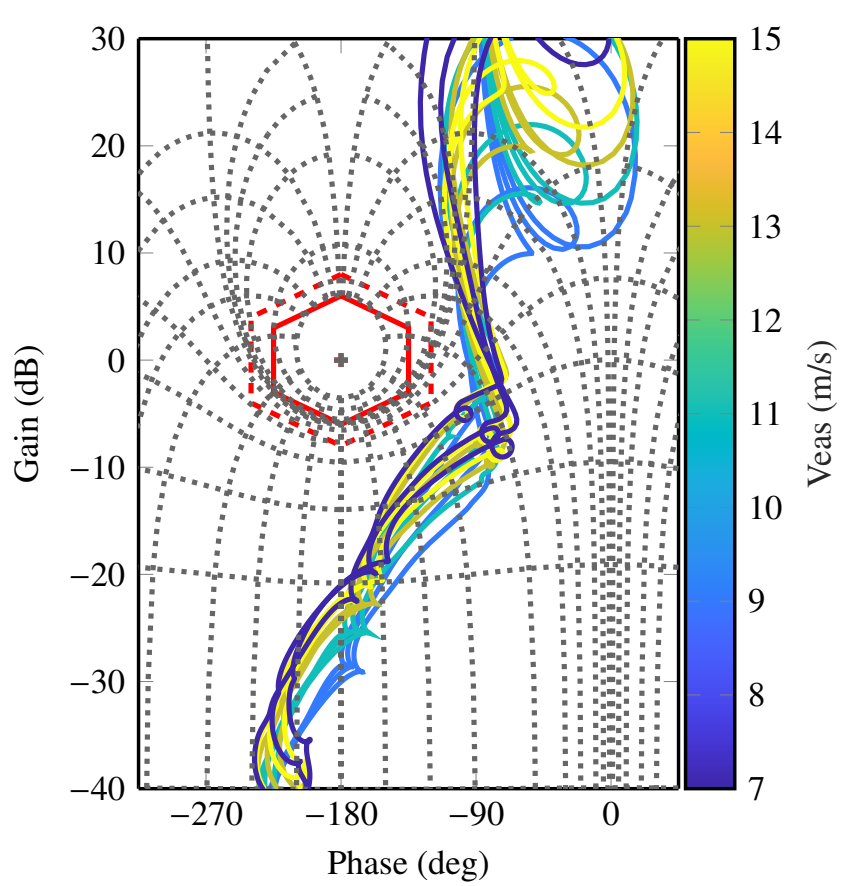

(b) Nichols chart.

Fig. 10 Step response of the optimized closed loop for the full envelope and Nichols chart showing the gain / phase margins for the pitch RCAH controller and longitudinal dynamics open loop model. 
The optimization results for the RCAH lateral controller are shown in Fig. 11 The result of the linear closed loop step response in Fig. 12. From these plots, the stability and performance requirements is cross-checked in order to confirm the success of the control law synthesis. As confirmed by the last two plots of Fig. 11, the system is stable with sufficient margins at all frequencies. The specified rise time for the roll attitude is met (Fig. 12) and the specified steady state error is met in the first plot of Fig.11] A detailed $\mathrm{V} \& \mathrm{~V}$ process is being developed for cross checking all requirements within the non-linear closed loop simulation (the detailed layout and results of the $\mathrm{V} \& \mathrm{~V}$ tool chain would go beyond the scope and will be addressed separately). Even though the available airspeed envelope is tight, it can be clearly seen that for all three parameters the available degree of freedom to introduce a scheduling over $V_{\text {eas }}$ seems necessary.
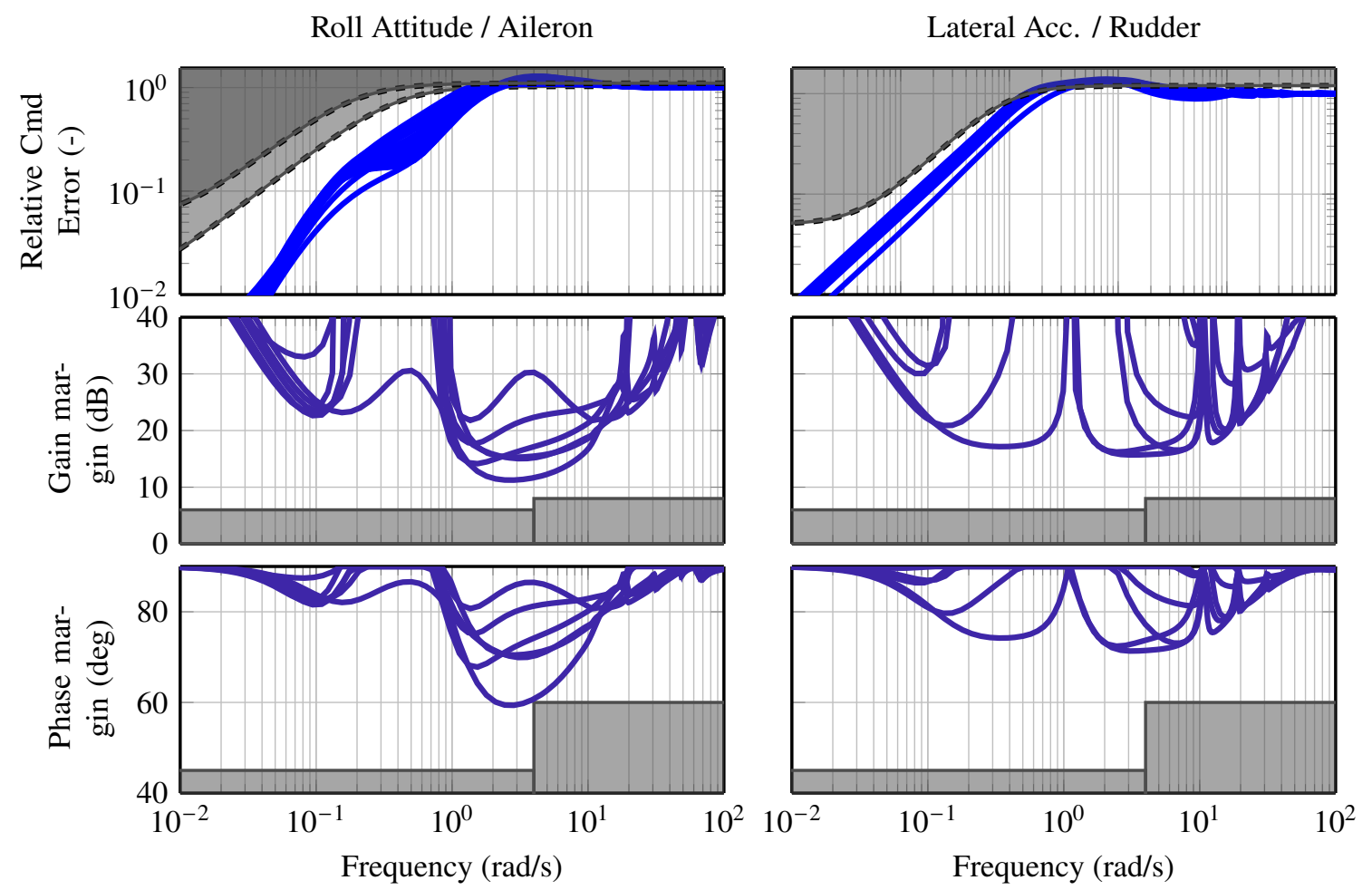

Fig. 11 Optimization results for the lateral control laws. First row: tracking requirement for bank attitude and lateral acceleration; Lower four plots: gain/phase margins on aileron and rudder channel.

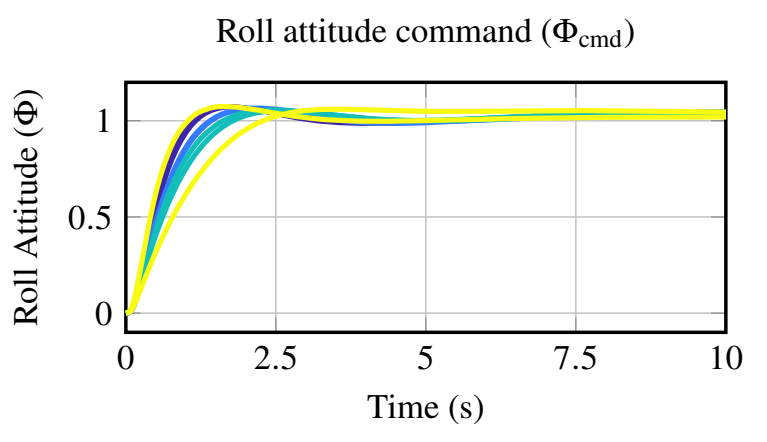

Fig. 12 Roll attitude step response to a roll attitude command for different airspeed values at FL0 / FL600. 


\section{Autopilot Design}

Similar to the inner loop control laws, the autopilot design is separated into the longitudinal and lateral axis of the aircraft. For implementing a longitudinal autopilot, the TECS [21] algorithm is chosen, as this shows promising results

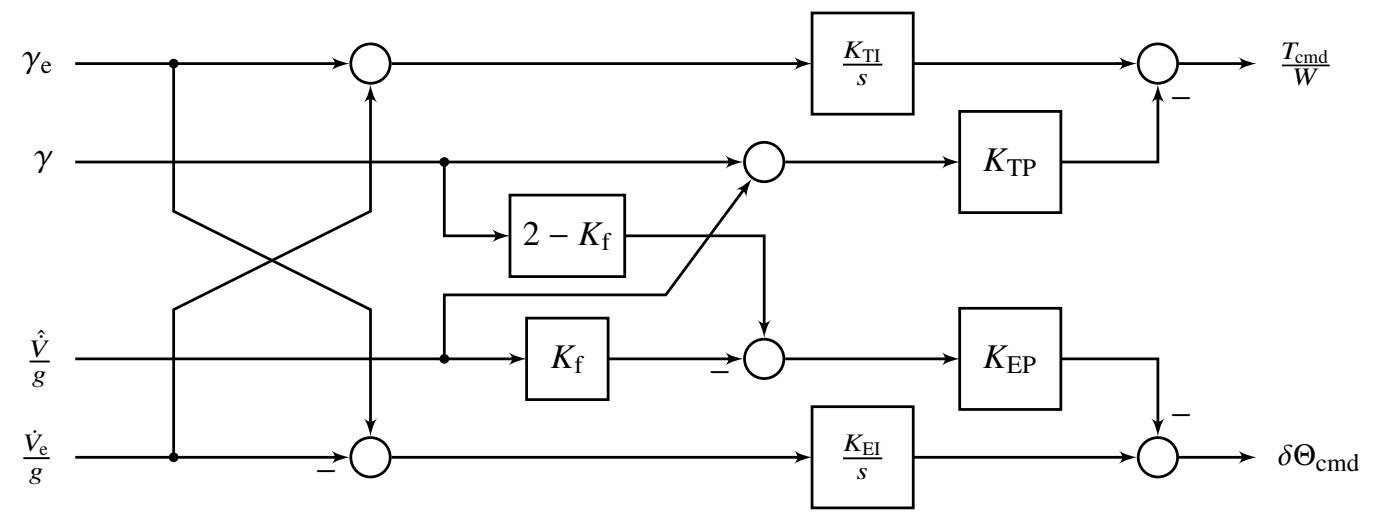

Fig. 13 Total Energy Control System (TECS) control architecture.

in previous HALE design project and is already proven in flight tests [22]. The TECS control law uses the principal of the total energy of the aircraft

$$
\begin{aligned}
E & =E_{\text {pot }}+E_{\text {kin }} \\
\dot{E} & =W \dot{h}+W V(V) / g \\
& \simeq W V\left(\gamma+\frac{\dot{V}}{g}\right)
\end{aligned}
$$

where a demand in energy change $(\dot{E})$ can directly be related to a thrust command, as shown in Eq. 10 , with the assumption that the increase in drag is relatively small compared to the thrust increase. With the specific total energy rate defined as $\dot{E}_{S}=\dot{E} /(W V)$ and the errors in flight path angle $\gamma$ and equivalent airspeed ( $\left.\mathrm{V}_{\text {eas }}\right)$, a proportional integral (PI) control law for the thrust can be defined in Eq. [15].

$$
\begin{aligned}
\Delta \dot{E}_{S} & =\left(\gamma_{\mathrm{cmd}}-\gamma\right)+\left(\dot{V}_{\mathrm{cmd}}-\dot{V}\right) / g \\
& =\left(\Delta T_{\mathrm{cmd}}-\Delta D\right) / W \simeq \Delta T_{\mathrm{cmd}} / W \\
\frac{\Delta T_{\mathrm{cmd}}}{W} & =K_{E I} \int \Delta \dot{E}_{S} d t-K_{E P} \dot{E}_{S}
\end{aligned}
$$

The specific energy distribution $\dot{D}_{s}$ between kinematic and potential energy rate is defined in Eq. 16.

$$
\begin{aligned}
\dot{D}_{S} & =-E_{\mathrm{S}, \mathrm{pot}}+E_{\mathrm{S}, \mathrm{kin}}=-\gamma+\frac{\dot{V}}{g} \\
\Delta \dot{D}_{S} & =\dot{D}_{S, \mathrm{cmd}}-\dot{D}_{S} \\
& =-\left(\gamma_{\mathrm{cmd}}-\gamma\right)+\frac{\left(\dot{V}_{\mathrm{cmd}}-\dot{V}\right)}{g}
\end{aligned}
$$

With the assumption that commanded energy distribution rate is proportional to the commanded pitch attitude, $\Delta \dot{D}_{S} \propto \Delta \Theta_{\mathrm{cmd}}$, the following control law can be derived for the control of the pitch inner loop:

$$
\Delta \Theta_{\mathrm{cmd}} \propto K_{D I} \int \Delta \dot{D}_{S} d t-K_{D P} \dot{D}_{S}
$$

The used TECS structure is depicted in Fig. 13. The proportional and integral gains for the specific energy rate in Eq. (15) and specific energy distribution Eq. (17) are tuned in order to achieve a bandwidth of $25 \%$ the inner loop bandwidth, together with gain / phase margin and disturbance rejection specifications shown in Tab.5. Note, that for 
the tuning of the TECS autopilot the closed loop function obtained in Sec. IV is used as plant G(s). In contrast to the preciously presented controller optimization, the fixed structure control system is tuned with a single set of gains for all operating points of the flight envelope. Fig. 14 shows the preliminary mentioned bandwidth specification and the resulting loop transfer and complimentary sensitivity functions for the flight envelope.

Table 5 TECS controller tuning specifications.

\begin{tabular}{lcc}
\hline Name & Acceptable Value & Frequency Region \\
\hline Airspeed bandwidth & $0.5 \mathrm{rad} / \mathrm{s}$ & - \\
Path angle bandwidth & $0.5 \mathrm{rad} / \mathrm{s}$ & - \\
Gain / Phase Margin & $6 \mathrm{~dB} / 45 \mathrm{deg}$ & \\
Dist. rejection pitch & $15 \mathrm{~dB}$ & {$[0 ; 0.01] \mathrm{rad} / \mathrm{s}$} \\
Dist. rejection thrust & $30 \mathrm{~dB}$ & {$[0 ; 0.01] \mathrm{rad} / \mathrm{s}$} \\
\hline
\end{tabular}
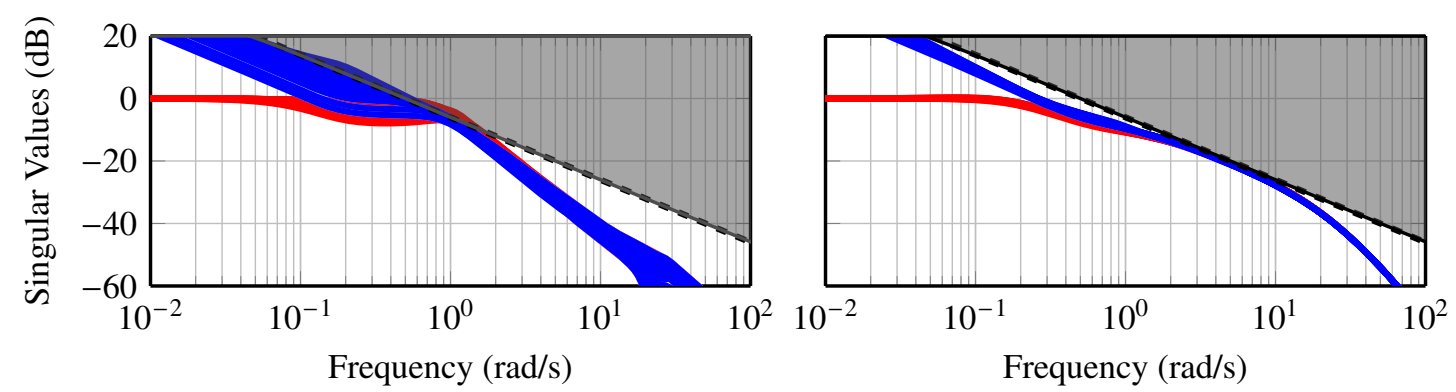

Fig. 14 Singular Values of the Loop Transfer functions $\mathrm{L}=$ GC (blue) and the complimentary sensitivity functions $T$ (red) for the full flight envelope. The left hand side plot shows the bandwidth of the pitch dynamics output, the right hand side shows the thrust output.

For operation, the TECS structure inherently supports two main modes which are interesting for operation of a HALE aircraft:

1) Speed by pitch mode: the flight path angle cross-feed to the energy distribution signal is switched off, as well as the thrust output. The pilot controls the thrust manually, whereas the airspeed is controlled by the autopilot via the energy distribution channel. In consequence, the pilot's throttle selection directly reflects on the flight path angle $\gamma$ whereas airspeed is maintained automatically. This mode can e.g. be used for maximum rate climbs or descents by setting the throttle manually to max. continuous climb, or idle respectively.

2) Decoupling for path and speed: this indicates the "conventional" TECS use, where both flight path and airspeed are controlled.

For the lateral autopilot, a structure consisting of proportional gains, as applied in standard transport aircraft [19], is chosen. This consists of a proportional gain on the heading / track error, as seen in Eqs. 20] and 21]. The result of this equation is fed to the lateral autopilot and inserted as bank angle command to the lateral inner loop. For flight path following, the before mentioned system is augmented in another cascade by a trajectory following command, which accepts the desired ground track, $\chi_{\text {track }}$ and the cross-track error $\Delta_{y}$ as inputs. Eq. 22) gives the resulting path command which then is used to calculate the path error $e_{\chi}$ in Eq. 21. The yaw gain can be tuned via root locus or loop-tuning in order to ensure sufficient gain / phase margins and a bandwidth of less than $.5 \mathrm{rad} / \mathrm{s}$ for the full envelope with a single yaw gain.

$$
\begin{gathered}
\Phi_{\mathrm{cmd}}=K_{\Psi} \frac{V_{\mathrm{tas}}}{g} e_{\Psi} \\
\Phi_{\mathrm{cmd}}=K_{\Psi} \frac{V_{\mathrm{tas}}}{g} e_{\chi} \\
\chi_{\mathrm{cmd}}=\frac{K_{y} \Delta_{y}}{V_{g}}+\chi_{\text {track }}
\end{gathered}
$$




\section{Control System Verification}

In order to demonstrate all presented features of the designed control law, a combined flight scenario including the path tracking of a holding pattern in combination with a step climb during part of the holding is simulated with the non-linear high fidelity model of the aircraft. The holding pattern is chosen, as it is a typical scenario for HALE aircraft during ascent and payload operation. For the simulation, the non-linear flexible aircraft model, high-fidelity actuator models, sensor models including delay and the presented flight controller are interconnected. The simulation results are shown in Fig. 15, with the longitudinal aircraft motion parameters on the left hand side, lateral motion variables on the right hand side. The first row visualizes the flown holding pattern (right) in combination with a step climb of $100 \mathrm{~m}$ (left). The black cross shows the reference point for the first leg. The climb is executed only during the crosswind section until the aircraft reaches the final altitude at the end of the downwind leg of the pattern. The second row of diagrams depicts the flight path angle with flight path angle command, as well as the course angle. It can be seen that both signals are tracked smoothly with their respective time constants and without any overshoot. The final row shows the equivalent airspeed. The TECS algorithm maintains airspeed with only satisfactory small deviations during the changes in flight path and bank. This overall simualtion finally shows that the developed flight control system meets the defined requirements and provides satisfactory flight though even more complex scenarios.
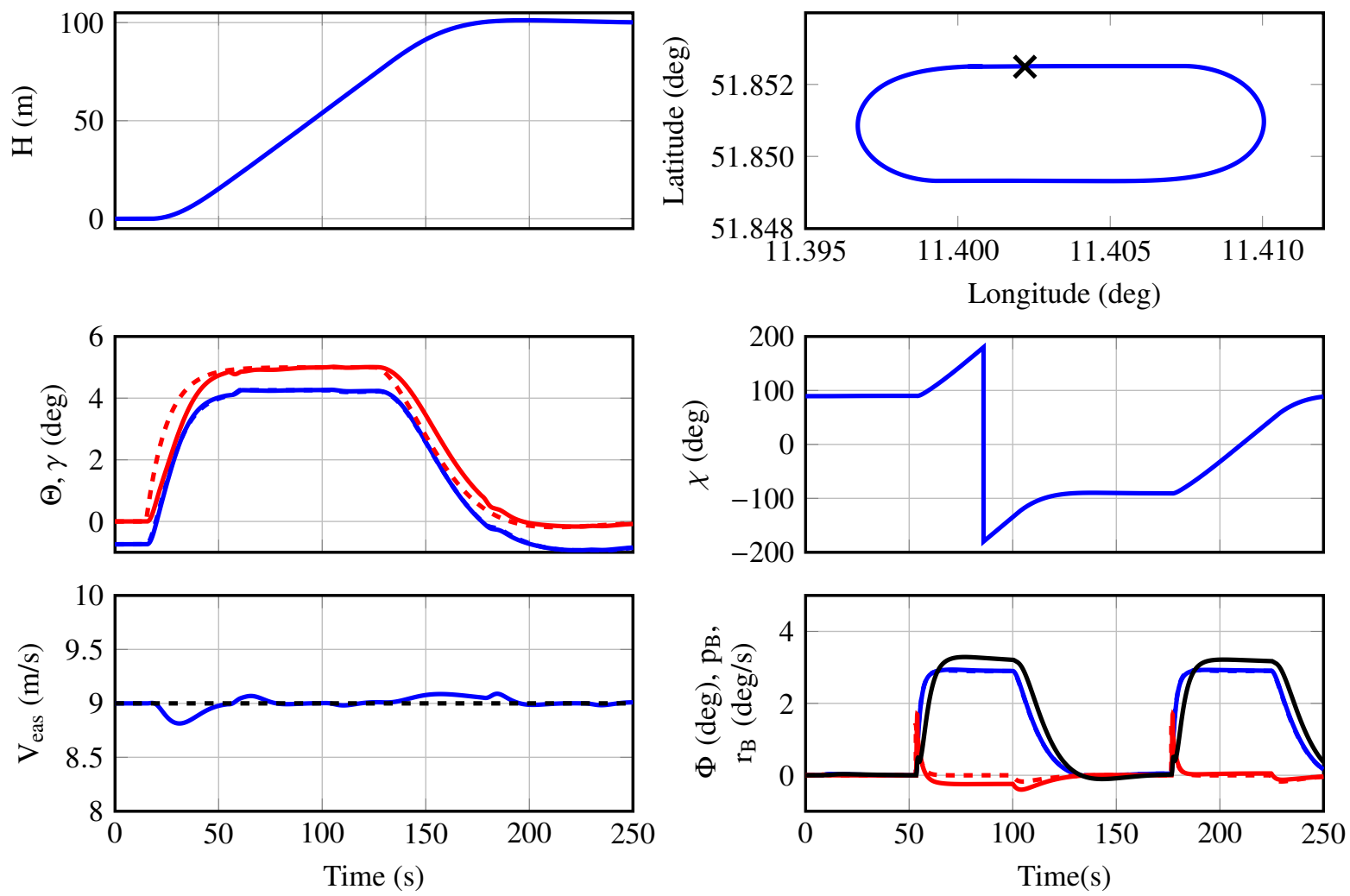

Fig. 15 Non-linear simulation results of a racetrack pattern combined with an step climb in altitude. Left hand side plots: altitude (top), pitch attitude (red) and flight path angle (middle), equivalent airspeed (bottom). Right hand side: ground track of holding pattern (top), course angle (middle), roll attitude(blue), roll rate (red), and turn rate(black). Dashed lines depict command values. 


\section{Conclusion}

In this paper, the requirements based design of a gain-scheduled flight control system as well as an autopilot for a high altitude long endurance aircraft is presented. The requirements are specifically tailored to the displayed aircraft configuration. A classical cascaded flight controller structure is adopted and the fixed control structure is tuned with a constraint optimization algorithm to meet the design requirements. Finally, a non-linear simulation is carried out to validate the performance of the control laws at one specific operating point. Future work will include the automated assessment of controller performance throughout the full flight envelope, as well as the robustness to disturbances (e.g. 1-cosine gusts, turbulence, model uncertainties) within the operational envelope.

\section{Acknowledgments}

The presented work is part of the German Aerospace Center's High Altitude Platform (HAP) project. Presented methods and results were partly developed within the scope of the project.

\section{References}

[1] Keidel, B., “Auslegung und Simulation von hochfliegenden, dauerhaft stationierbaren Solardrohnen,” Ph.D. thesis, TU München, 2000.

[2] Noll, T. E., Brown, J. M., Perez-Davis, M. E., Ishmael, S. D., Tiffany, G. C., and Gaier, M., "Investigation of the Helios Prototype Aircraft Mishap,” techreport, NASA, 2004.

[3] Airbus, "Zephyr S set to break aircraft world endurance record,", 07/2018. URL https://www . airbus.com/defence/uav/ zephyr.html\#introduction

[4] "In-flight break-up involving Airbus Zephyr unmanned aerial vehicle," Tech. rep., Australian Transport Safety Bureau (ATSB), $09 / 2020$.

[5] Hasan, Y. J., Roeser, M. S., Hepperle, M., Niemann, S., Voß, A., Handojo, V., and Weiser, C., "Flight Mechanical Design and Analysis of a Solar-Powered High-Altitude Platform," 69. Deutscher Luft- und Raumfahrtkongress, 2020.

[6] Voß, A., Handojo, V., Weiser, C., and Niemann, S., "Preparation of Loads and Aeroelastic Analyses of a High Altitude, Long Endurance, Solar Electric Aircraft,” Aerospace Europe Conference, 2020.

[7] DLR, "DLR develops an unmanned stratospheric aircraft,", 2021. URL https://www.dlr.de/content/en/articles/ news/2021/02/20210421_dlr-develops-an-unmanned-stratospheric-aircraft.html

[8] Nikodem, F., and Bierig, A., "DLR HAP - Herausforderungen in der Entwicklung der Höhenplattform und ihrer Anwendungen," Deutscher Luft- und Raumfahrtkongress (DLRK), 2020.

[9] Kier, T. M., and Hofstee, J. W., "Varloads - Eine Simulationsumgebung zur Lastenberechnung eines voll Fĺexiblen, Freifliegenden Flugzeugs," Deutscher Luft- und Raumfahrtkongress (DLRK), 2004.

[10] Kier, T., Looye, G., and Hofstee, J., "Development of Aircraft Flight Loads Analysis Models with Uncertainties for Pre-design Studies," Proc. of IFASD 2005, International Forum on Aroelasticity and Structural Dynamics, 2005.

[11] Braun, S., "Structurally Constrained Lateral Control of a Diamond-Shaped Unmanned Aircraft," Ph.D. thesis, Technische Universität München, 2019.

[12] Köthe, A., "Flight Mechanics and Flight Control for a Multibody Aircraft," Ph.D. thesis, Technische Universität Berlin, 2019.

[13] Pusch, M., Ossmann, D., and Luspay, T., "Structured Control Design for a Highly Flexible Flutter Demonstrator," Aerospace. Multidisciplinary Digital Publishing Institute (MDPI), 2019.

[14] “AS94900 Vehicle Management Systems - General Specification Guide for Flight Control Function, Design, Installation and Test of Piloted Military Aircraft,”, 2018.

[15] “ARP94910 Vehicle Management Systems - Specification Guide for Flight Control Design, Installation and Test of Military Unmanned Aircraft,", 2012.

[16] Joos, H.-D., Bals, J., Looye, G., Schnepper, K., and Varga, A., "A multi-objective optimisation-based software environment for control systems design," IEEE International Conference on Control Applications and International Symposium on Computer Aided Control Systems Design, 2002, pp. 7-14. 
[17] Apkarian, P., Gahinet, P., and Buhr, C., "Multi-model, multi-objective tuning of fixed-structure controllers," 2014 European Control Conference (ECC), 2014, pp. 856-861.

[18] Apkarian, P., Dao, M., and Noll, D., "Parametric Robust Structured Control Design," IEEE Transactions on Automatic Control, Vol. 60, 2015, pp. 1857-1869. doi: https://doi.org/10.1109/TAC.2015.2396644.

[19] Brockhaus, R., Alles, W., and Luckner, R., Flugregelung, Springer, 2001.

[20] Andry, A. N., Shapiro, E. Y., and Chung, J., "Eigenstructure Assignment for Linear Systems," IEEE Transactions on Aerospace and Electronic Systems, Vol. AES-19, No. 5, 1983, pp. 711-729. doi: https://doi.org/10.1109/TAES.1983.309373.

[21] Lambregts, A. A., “TECS Generalized Airplane Control System Design - An Update,” Advances in Aerospace Guidance, Navigation and Control, Control,, Springer, 2013, pp. 503-534.

[22] Kastner, N., and Looye, G., "Generic TECS based autopilot for an electric high altitude solar powered aircraft," Proceedings of the EuroGNC 2013, 2nd CEAS Specialist Conference onGuidance, Navigation \& Control, 2013, pp. 1324-1343. 